\title{
ORIENTAÇÃO PARA O MERCADO E HABILIDADES DE MARKETING INTERNACIONAL: UM ESTUDO ACERCA DA INFLUÊNCIA SOBRE O DESEMPENHO INTERNACIONAL
}

\author{
GUIDANCE FOR MARKET \\ AND INTERNATIONAL MARKETING SKILLS: \\ A STUDY ABOUT THE INFLUENCE ON THE \\ INTERNATIONAL PERFORMANCE
}

\author{
Data de submissão: $13 / 11 / 2016$ \\ Aceite: 30/06/2017 \\ Maria Carolina Serpa Fagundes de Oliveira ${ }^{1}$ \\ Ivanete Schneider Hahn ${ }^{2}$ \\ Maríndia Brachak dos Santos ${ }^{3}$ \\ Gabriela Almeida de Souza ${ }^{4}$ \\ Daiane Cardozo Campos ${ }^{5}$ \\ Natália Pavanelo Pivetta ${ }^{6}$
}

\section{RESUMO}

Diante das permanentes transformações sociais e econômicas e em virtude da constante busca por maior competitividade, existe atualmente uma necessidade de que as empresas voltem sua atenção para os aspectos mercadológicos, com o fim de buscarem maior excelência na geração de valor aos consumidores. Tal configuração, quando destinada a uma realidade de comércio internacional, pode representar os definidores de crescimento e consolidação organizacional. Por isso, ao observar tal ordenação e necessidade, o presente trabalho buscou analisar a influência da orientação para o mercado internacional e das habilidades de marketing internacional sobre o desempenho internacional das organizações. A coleta de dados ocorreu juntamente a 53 EBTs brasileiras localizadas em ambientes de inovação. Tais dados foram analisados por meio de técnica estatística multivariada, permitindo contatar que o desempenho internacional pode ser influenciado pela orientação para o mercado internacional.

Palavras-chave: internacionalização, marketing internacional, desempenho internacional.

\footnotetext{
1 Possui graduação em Administração pela Universidade Federal de Santa Maria. Mestrado em Administração pela Universidade Federal de Santa Maria. Santa Maria. Rio Grande do Sul. Brasil. E-mail: mc.sfagundes@gmail.com

2 Possui Graduação em Administração pela Faculdade de Itapiranga (FAI). Mestre e Doutoranda em Administração pelo Programa de Pós-graduação em Administração da Universidade Federal de Santa Maria. Atua como docente Universidade Alto Vale do Rio do Peixe (UNIARP) e no SENAC Santa Catarina. Caçador. Santa Catarina. Brasil. E-mail: ivischneider@hotmail.com

3 Possui Graduação em Ciências Contábeis pela Universidade Comunitária Regional de Chapecó. Mestrado em Administração pelo PPGA/UFSM. Atualmente é Doutoranda em Administração pelo Programa de Pós-Graduação em Administração PPGA/UFSM - Linha de Pesquisa: Estratégia em Organizações. Também é Professora na área de Contabilidade no Colégio Politécnico da Universidade Federal de Santa Maria. Santa Maria. Rio Grande do Sul. E-mail: marindiabrachak@gmail.com

4 Possui Graduação em andamento em Administração na Universidade Federal de Santa Maria. Santa Maria. Rio Grande do Sul. E-mail: gabialmeida06@hotmail.com

5 Possui Graduação em Administração pela Universidade Federal de Santa Maria. Santa Maria. Rio Grande do Sul. E-mail: daiane.adm.93@gmail.com 6 Possui graduação em Administração pela Universidade Federal de Santa Maria. Atualmente é mestranda em Administração na linha de Estratégia em Organizações, pelo Programa de Pós-Graduação em Administração da Universidade Federal de Santa Maria. Santa Maria. Rio Grande do Sul. Brasil. E-mail: nataliappivetta@gmail.com
} 


\begin{abstract}
In the face of ongoing social and economic changes and because of the constant search for greater competitiveness, there is currently a need for companies to turn their attention to marketing aspects, in order to seek greater excellence in the generation of value to consumers. This configuration, when allocated to a reality of international trade, can represent the growth of defining and organizational consolidation. Therefore, when observing this ordinance and need, this study aimed to analyze the influence of orientation to the international market and international marketing skills on the international performance of organizations. A collection of data was carried out in conjunction with 53 Brazilian EBTs located in innovation environments. Such data and analyzes using multivariate statistical technique and with the obtained results, it was contacted that the international performance can be influenced by the orientation to the international market.
\end{abstract}

Keywords: internationalization, international marketing, international performance.

\title{
1 INTRODUÇÃO
}

Com a maior abertura comercial e o advento da tecnologia de comunicação, atentar para o mercado internacional pode significar uma estratégia de crescimento e, em certos casos, de sobrevivência para as empresas. De acordo com Moura e Honório (2012), organizações de diferentes portes e setores estão, cada vez mais, almejando atuar no mercado internacional, muitas vezes lançando mão das mais diferentes alternativas para o alcance do almejado objetivo.

Vista pelo prisma da estratégia, a internacionalização influencia as empresas no tocante aos seus aspectos sistêmicos e de longo prazo, tendo em vista uma imagem organizacional de complexidade e complementariedade. Por isso, atentar para qualquer elemento que venha a influir nos negócios internacionais torna-se significativamente importante para a gestão da empresa como um todo.

Dois desses aspectos estão ligados às funções de marketing e relacionam-se a uma maior orientação da empresa em voltar-se para o mercado internacional e às habilidades de marketing internacional de que as organizações possam se valer. Com isso, atenta-se para os aspectos que tangem à habilidade empresarial em entender a ação de seus concorrentes, bem como em conseguir coordenar as ações internacionais entre as áreas funcionais da empresa e as habilidades da empresa em criar valor para os clientes internacionais por meio de uma efetiva segmentação e avaliação d as ferramentas de marketing (RIBEIRO, 2012; KNIGHT; KIM, 2009).

No entanto, ainda existem algumas lacunas no que concerne à capacidade dos fatores relacionados ao marketing em influenciar o desempenho internacional. Segundo Gnizy (2016), as evidências empíricas acerca de tal influência ainda são limitadas e, em certas situações, contraditórias. $\mathrm{O}$ autor evidencia, ainda, a existência de estudos que entendem que as funções de marketing contribuem de maneira direta para o desempenho organizacional, enquanto que outros não comprovam semelhante relação.

Ademais, de acordo com Martin e Javalgi (2016), as economias de mercado, incluindo as de diversos países emergentes da América Latina, são consideradas como novos motores do crescimento econômico (LEDERMAN et al., 2014). No entanto, os autores acreditam que a maioria das pesquisas sobre internacionalização e desempenho ainda são limitadas em meio a essa conjuntura, sendo centradas apenas em multinacionais já estabelecidas (CARNEIRO; BRENES, 2014; FLEURY; FLEURY, 2014).

Por isso, ao atentar para as lacunas apresentadas, este estudo visa analisar, diante de um contexto de empresas internacionalizadas, a influência da orientação para o mercado internacional e as habilidades de marketing internacional sobre o desempenho internacional das 
organizações. Para isso, com o uso de análise quantitativa, buscou-se identificar se tais aspectos relacionados ao âmbito do marketing internacional podem influenciar a avaliação do desempenho provindo da comercialização no mercado externo.

De maneira a atender ao objetivo proposto pelo presente estudo, este artigo é composto de quatro seções. Além desta introdução, as seções seguintes discorrem acerca do embasamento conceitual e teórico que sustenta a pesquisa e as hipóteses, dos aspectos metodológicos que nortearam a realização da pesquisa e dos principais resultados alcançados.

\section{FUNDAMENTAÇÃO TEÓRICA}

Com o objetivo de explanar as bases teóricas que sustentam o presente estudo, nesta seção é apresentada a fundamentação teórica que apoia as hipóteses da pesquisa.

\subsection{ORIENTAÇÃO PARA O MERCADO INTERNACIONAL}

A orientação para o mercado é um constructo já muito utilizado, seja por sua capacidade de criar vantagem competitiva, seja por constituir-se em uma estratégia para o desenvolvimento de mercados internacionais (BARBOSA; AYALA, 2014). Essa orientação pode ser vista como a ênfase organizacional no sucesso em nível mundial ao invés do sucesso país-a-país e constitui parte da cultura organizacional (ZOU; CAVUSGIL, 2002).

Segundo Dong et al. (2016), a orientação para o mercado enfatiza atividades que estão relacionadas com a geração de inteligência de mercado, a disseminação dessa inteligência em todos os departamentos e a capacidade de resposta ao mercado (KOHLI; JAWORSKI, 1990). Tal orientação deriva da área de marketing internacional e caracteriza as empresas que buscam identificar as melhores oportunidades no mercado internacional e reduzir os riscos associados à dinâmica empresarial, tornando-se, assim, mais competitivas (BARBOSA; AYALA, 2014).

Uma empresa orientada para o mercado, de forma proativa e sistemática, consegue avaliar melhor a inteligência desse mercado a respeito de clientes, concorrentes, governo, tecnologia e outros aspectos ambientais (HE; WEI, 2011) que influenciem seus negócios e possam ser forças impulsionadoras de oportunidades. Por isso, quando utilizada de forma estratégica, a orientação para o mercado produz valor para os clientes, habilidade que pode ser considerada de rara e difícil imitação (NARVER; SLATER; MACLACHLAN, 2000), podendo ser usada como uma fonte de vantagem competitiva sustentável que permitirá às empresas a superação de seus concorrentes de mercado (LIAO et al., 2011).

Tal entendimento é complementado por Narver, Slater e Maclachlan (2000), ao indicarem que a orientação para o mercado é um dos elementos que levam a um desempenho positivo, assim como outros fatores específicos dos negócios (custos e tamanho) e fatores de mercado (crescimento, concentração, barreiras de entrada, poder dos compradores e vendedores e mudança tecnológica). Por isso, a orientação para o mercado, assim como comprovado por averiguações empíricas recentes (PAIM; LARA; CHRISTINO, 2011), assume uma significativa importância para o desempenho organizacional de uma empresa internacionalizada (MORAIS; SOARES, 2016). Esse argumento se sustenta no fato de que as organizações voltadas para o mercado, ou seja, aquelas que acompanham as necessidades e preferências dos clientes e reagem a elas, podem melhor satisfazer seus clientes e, portanto, apresentam melhor desempenho (JAWORSKI; KOHLI, 2006).

Segundo García et al. (2012), o avanço das empresas em sua internacionalização está fortemente associado às orientações e motivações diretivas que estabelecem determinada lógica na 
organização (FILATOTCHEV et al., 2009). Por isso, ao facilitar o conhecimento sobre os clientes e, consequentemente, sobre o desenvolvimento de produtos, a orientação voltada para o mercado pode gerar resultados para o desempenho internacional das empresas (KNIGHT; CAVUSGIL, 2004).

Com base nas afirmações apresentadas, sustenta-se a seguinte hipótese: internacional.

$\mathbf{H}_{1}$ : a orientação para o mercado internacional influencia positivamente o desempenho

\subsection{HABILIDADES DE MARKETING INTERNACIONAL}

As capacidades de marketing podem ser consideradas as habilidades e competências processadas em uma firma que ajudam a entender as mudanças que ocorrem nos mercados comerciais, com vistas a perceber quais delas não são mais eficientemente capazes de serem utilizadas (DAY, 1994). Para isso, é necessário valer-se de um variado grupo de habilidades, tais como capacidade de sentir e pesquisar o mercado, de criar laços com o mercado e de gerenciar o relacionamento com os clientes (RIPOLLÉS; BLESA, 2012).

Conforme apontado por Dalmoro (2009), com o processo de maior abertura dos mercados ocorrido há mais de duas décadas, a competição surge não apenas de concorrentes tradicionais ou de novos entrantes em determinados setores industriais, mas também a partir da desintegração de barreiras de acesso a mercados anteriormente isolados (TAPSCOTT; CASTON, 1995). Com isso, a transformação interna da empresa que pretende se dedicar à comercialização no exterior inclui uma modificação da sua própria cultura, uma vez que a comercialização no exterior não representa somente o aproveitamento de uma oportunidade pontual, pois deverá fundamentalmente fazer parte de uma estratégia global, que é efêmera, sobretudo no que tange ao crescimento da empresa (PALACIOS; SOUSA, 2004).

Por isso, a decisão de realizar atividades em outros países obriga as empresas a tomarem uma série de decisões em termos de estratégias de marketing para cada um dos locais em que atuam ou vão passar a atuar (LINDON et. al., 2000), o que exige a concepção de habilidades próprias de resposta à complexidade do mercado internacional e à matização de seus principais concorrentes. A julgar pelo entendimento da orientação para o mercado como uma capacidade organizacional, Zhou (2007) afirma que muitas empresas recém-internacionalizadas fazem um salto mais cedo para o mercado internacional por causa dessas capacidades próprias.

Os riscos de expansão empresarial nos mercados internacionais são grandes e de difícil previsão (PALACIOS; SOUSA, 2004). Em meio a tal realidade, o atual momento de competitividade mais acirrada leva as empresas a desenvolverem conceitos de marketing para antecipar, responder e capitalizar as mudanças no mercado, proporcionando melhor desempenho empresarial (NETO et al., 2013).

Para Song, Nason e Benedetto (2008), a capacidade de marketing internacional pode ter um papel fundamental para o sucesso das atividades desempenhadas no ambiente externo, em razão de que a habilidade de adquirir conhecimento, atrelada ao atendimento das necessidades dos clientes e à bem-sucedida venda de produtos, pode ser observada como importante fator para a determinação da prosperidade organizacional, mesmo para as pequenas e médias empresas (BRETTEL; ENGELEN; HEINEMANN, 2009; ZAHRA; IRELAND; HITT, 2000).

Essa definição encontra respaldo em Cavusgil e Zou (1994), ao defenderem que o desempenho internacional é influenciado pelas estratégias de marketing, na medida em que existem alguns fatores que contribuem para esse fim, como produto oferecido, contato realizado com os distribuidores ou agentes estrangeiros, estratégia de promoção utilizada e preço aplicado 
na operação, além de determinadas características da firma, como experiência e comprometimento internacional (MOURA; HONÓRIO, 2012). Isso ocorre em virtude do fato de que fortes ações de marketing podem acelerar o processo organizacional, assim como a produção de novos produtos, funcionando, consequentemente, como um drive de desempenho (HOMBURG et al., 2015) e sendo, por isso, concebidas como influenciadores direto do desempenho organizacional (GNIZY, 2016; AUH; MERLO, 2012).

Com base nas afirmações apresentadas, sustenta-se a seguinte hipótese:

$\mathbf{H}_{2}$ : a habilidade em marketing internacional influencia positivamente o desempenho internacional.

\section{PROCEDIMENTOS METODOLÓGICOS}

O presente estudo define-se por seu caráter quantitativo. Realizou-se mediante uma pesquisa descritiva e de acordo com a configuração conceitual desenvolvida pelos modelos de pesquisa de Ribeiro, Oliveira Jr. e Borini (2012) e Gerschewski, Rose e Lindsay (2015), que versam sobre os fatores abordados na pesquisa. O modelo conceitual utilizado é apresentado a seguir, na Figura 1.

Figura 1 - Modelo de pesquisa

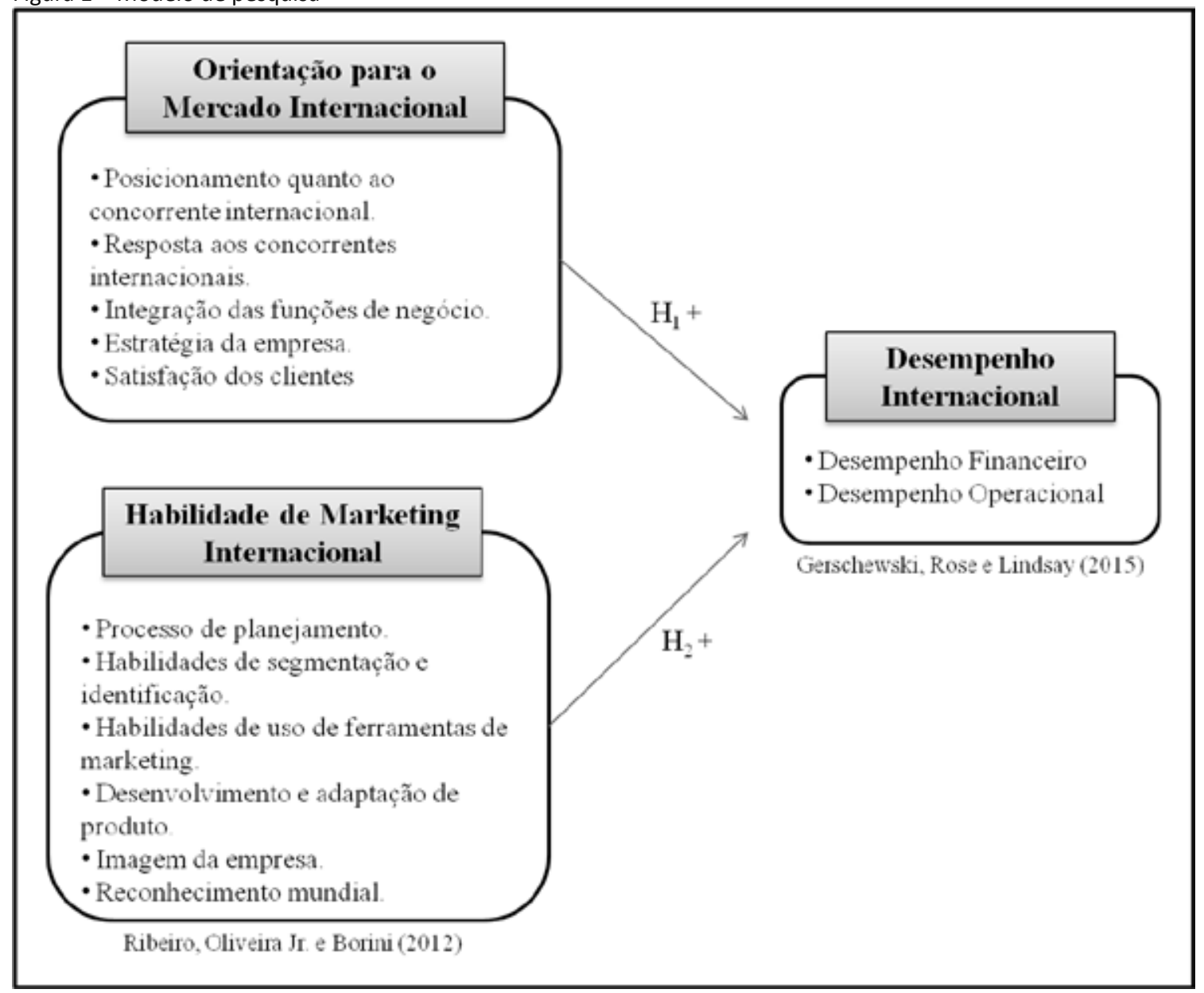

Fonte: elaborado pelos autores com base em Ribeiro, Oliveira Jr. e Borini (2012) e Gerschewski, Rose e Lindsay (2015). 
Para a coleta de dados, utilizou-se um questionário elaborado com base nos modelos que embasam o estudo e composto de três dimensões (orientação para o mercado internacional, habilidade de marketing internacional e desempenho internacional) que utilizam o mesmo tipo de escala. O constructo de desempenho internacional, desenvolvido por Gerschewski, Rose e Lindsay (2015), foi submetido aos procedimentos de tradução e back translation realizados por dois tradutores independentes, conhecedores do idioma e cientes dos objetivos da pesquisa. Posteriormente, a validação foi realizada por meio de juízes e acadêmicos da área (MALHOTRA, 2001), sendo as variáveis do constructo consideradas adequadas por três professores do Programa de Pós Graduação em Administração (PPGA) da Universidade Federal de Santa Maria (UFSM).

$O$ instrumento de coleta de dados é formado por 23 variáveis medidas em escala Likert de 5 pontos (Quadro 1), além de conter sete variáveis acerca do perfil das empresas que compõem a amostra estudada.

Quadro 1 - Operacionalização das variáveis

\begin{tabular}{|c|c|}
\hline Dimensão & Mensuração \\
\hline $\begin{array}{c}\text { Orientação para o } \\
\text { mercado internacional }\end{array}$ & $\begin{array}{l}5 \text { itens - escala do tipo Likert de } 5 \text { pontos } \\
\text { Concordância com: } \\
\text { - posicionamento quanto ao concorrente internacional; } \\
\text { - resposta aos concorrentes internacionais; } \\
\text { - integração das funções de negócio; } \\
\text { - estratégia da empresa; } \\
\text { - satisfação dos clientes. }\end{array}$ \\
\hline $\begin{array}{l}\text { Habilidade de marketing } \\
\text { internacional }\end{array}$ & $\begin{array}{l}6 \text { itens - escala do tipo Likert de } 5 \text { pontos } \\
\text { Concordância com: } \\
\text { - processo de planejamento; } \\
\text { - habilidades de segmentação e identificação; } \\
\text { - habilidades de uso de ferramentas de marketing; } \\
\text { - desenvolvimento e adaptação de produto; } \\
\text { - imagem da empresa; } \\
\text { - reconhecimento mundial. }\end{array}$ \\
\hline Desempenho internacional & $\begin{array}{l}12 \text { itens - escala do tipo Likert de } 5 \text { pontos } \\
\text { Grau de satisfação com: } \\
\text { - vendas internacionais; } \\
\text { - crescimento das vendas internacionais; } \\
\text { - rentabilidade internacional; } \\
\text { - retorno sobre o investimento (ROI); } \\
\text { - participação nos mercados internacionais; } \\
\text { - introdução de novos produtos/serviços no mercado interna- } \\
\text { cional; } \\
\text { - parcela de mercado dos novos produtos/serviços internacio- } \\
\text { nais; } \\
\text { - quantidade de novos produtos/serviços de sucesso no mer- } \\
\text { cado internacional; } \\
\text { - presença global. }\end{array}$ \\
\hline
\end{tabular}

Fonte: elaborado pelos autores com base em Ribeiro, Oliveira Jr. e Borini (2012) e Gerschewski, Rose e Lindsay (2015).

A população da pesquisa foi composta pelas Empresas de Base Tecnológica (EBTs), localizadas no Brasil, que desenvolvem atividades internacionais e se encontram em algum ambiente de inovação, como incubadoras tecnológicas, parques tecnológicos, aceleradoras de startups e clusters industriais. As EBTs são organizações pequenas que demonstram força na tomada de riscos, conduta inovadora e comportamentos proativos nas fases iniciais da sua fundação (LI; QIAN; QIAN, 2012). Para essas organizações, a expansão internacional representa uma oportunidade de 
crescimento e de criação de valor, pois, ao entrarem em mercados internacionais, geralmente, aumentam a sua experiência tecnológica e de mercado, melhoram o seu desempenho e, muitas vezes, tornam-se mais inovadoras (ENGELMAN; ZEN; FRACASSO, 2015).

O procedimento de coleta de dados ocorreu de forma eletrônica, com a utilização do sistema Survey Monkey, por meio do qual se efetuou o envio dos questionários. Já para o tratamento dos dados, como tabulação e análise, foram utilizados os softwares Microsoft Excel 2010 e Statistical Package for the Social Sciences (SPSS) versão 21.

Para a efetivação das análises estatísticas, foi realizada a preparação da matriz de entrada dos dados, em que foram verificados missing values, outliers e a normalidade dos dados. Devido à normalidade dos dados, efetuaram-se as análises descritivas das variáveis observáveis (frequência, média e desvio-padrão) e foram adotadas estatísticas multivariadas, de modo a testar as hipóteses do estudo.

\section{ANÁLISE E DISCUSSÃO DOS RESULTADOS}

Para o alcance do objetivo proposto - analisar a influência da orientação para o mercado internacional e das habilidades de marketing internacional sobre o desempenho internacional -, foram investigadas 53 EBTs brasileiras, localizadas nos estados de São Paulo, Rio Grande do Sul, Santa Catarina, Minas Gerais, Rio de Janeiro, Paraná, Pernambuco, Paraíba e Bahia e no Distrito Federal. Em sua maioria, as empresas participantes da pesquisa atuam no mercado há, em média, 12 anos, tendo adotado o padrão de Born Global de internacionalização. Pela quantidade de funcionários, caracterizam-se predominantemente como de micro e pequeno porte. Além disso, iniciaram suas atividades internacionais por meio de comércio eletrônico e comercializam, em maior número, com clientes da América do Norte.

\subsection{ANÁLISE FATORIAL}

Para a análise fatorial, foram consideradas duas dimensões do modelo de Ribeiro, Oliveira Jr. e Borini (2012) e uma dimensão do modelo de Gerschewski, Rose e Lindsay (2015), medidas por meio de escala do tipo Likert, com vistas a resumir os dados, para a obtenção de dimensões latentes que descrevam os dados em um número menor de conceitos do que as variáveis individuais originais (HAIR et al., 2009). Ademais, utilizou-se a técnica de análise dos componentes principais e realizaram-se testes para a verificação da adequação da análise fatorial - teste para a apuração do índice de Kaiser-Meyer-Olkin (KMO) e teste de esfericidade de Bartlett. Com isso, foram obtidos índices $\mathrm{KMO}$ superiores a 0,60 e teste de Bartlet com significâncias inferiores a 0,001 , o que indica a adequação dos dados para a realização de tal análise. O primeiro fator, designado de orientação para o mercado internacional, representa as habilidades da empresa em entender a ação dos concorrentes e conseguir coordenar as ações internacionais entre suas áreas funcionais. Nessa categoria, foram mantidas quatro variáveis, com cargas acima de 0,701 e variância explicada de 62,304\%. Nesse conjunto de dados, a variável MI1 (Em nossa empresa, frequentemente, discutem-se os pontos fortes e fracos de nosso principal concorrente internacional) foi excluída por apresentar comunalidade de 0,489, ou seja, abaixo de 0,50.

No segundo fator, sobre habilidades de marketing internacional, mantiveram-se todas as variáveis do modelo, pois estas apresentaram cargas fatoriais entre 0,729 e 0,869, além de uma variância explicada de $64,161 \%$. Esse fator trata das habilidades empresariais em criar valor para os clientes internacionais, por meio de uma efetiva segmentação e avaliação das ferramentas de marketing. Os valos referentes à análise são apresentados na Tabela 1. 
Tabela 1 - Análise fatorial dos fatores internos da empresa

\begin{tabular}{|c|c|c|c|c|c|}
\hline Fator & Variável & $\begin{array}{c}\text { Carga } \\
\text { fatorial }\end{array}$ & $\begin{array}{l}\text { Variância } \\
\text { explicada }\end{array}$ & KMO & $\begin{array}{l}\text { Alpha de } \\
\text { Cronbach }\end{array}$ \\
\hline \multirow{4}{*}{$\begin{array}{l}\text { Orientação } \\
\text { para o merca- } \\
\text { do internacio- } \\
\text { nal }\end{array}$} & $\begin{array}{l}\text { MI2 - Se um concorrente lançou uma cam- } \\
\text { panha intensiva orientada para os nossos } \\
\text { clientes internacionais, implementamos } \\
\text { uma resposta imediatamente. }\end{array}$ & 0,701 & \multirow{4}{*}{$62,304 \%$} & \multirow{4}{*}{0,719} & \multirow{4}{*}{0,794} \\
\hline & $\begin{array}{c}\text { MI3 - Nossas funções de negócio (por } \\
\text { exemplo, comercialização/vendas, fi- } \\
\text { nanças e produção) são integradas para } \\
\text { atender às necessidades do mercado in- } \\
\text { ternacional. }\end{array}$ & 0,742 & & & \\
\hline & $\begin{array}{l}\text { MI4 - A estratégia da empresa é baseada } \\
\text { em nossa compreensão das necessidades } \\
\text { dos clientes internacionais. }\end{array}$ & 0,807 & & & \\
\hline & $\begin{array}{c}\text { MI5 - A satisfação dos clientes internacio- } \\
\text { nais com nossos produtos ou serviços é } \\
\text { medida sistematicamente. }\end{array}$ & 0,844 & & & \\
\hline \multirow{6}{*}{$\begin{array}{l}\text { Habilidades de } \\
\text { marketing in- } \\
\text { ternacional }\end{array}$} & $\begin{array}{l}\text { MI6 - Processo de planejamento de } \\
\text { marketing internacional. }\end{array}$ & 0,847 & \multirow{6}{*}{$64,161 \%$} & \multirow{6}{*}{0,807} & \multirow{6}{*}{0,886} \\
\hline & $\begin{array}{l}\text { MI7 - Habilidade para segmentar e identi- } \\
\text { ficar nichos de mercado no exterior. }\end{array}$ & 0,784 & & & \\
\hline & $\begin{array}{l}\text { MI8 - Habilidade para usar ferramentas de } \\
\text { marketing para diferenciar o produto (de- } \\
\text { sign, formação de preço e publicidade). }\end{array}$ & 0,758 & & & \\
\hline & $\begin{array}{l}\text { MI9 - Desenvolvimento e adaptação do } \\
\text { produto no mercado internacional. }\end{array}$ & 0,729 & & & \\
\hline & MI10 - Imagem da empresa & 0,809 & & & \\
\hline & $\begin{array}{l}\text { MI11 - Reconhecimento mundial do pro- } \\
\text { duto/serviço. }\end{array}$ & 0,869 & & & \\
\hline
\end{tabular}

Fonte: dados da pesquisa.

Com os resultados obtidos pelo cálculo do Alpha de Cronbach, observa-se que os dois fatores utilizados apresentam consistência interna adequada, ao obterem valores de alpha de 0,794 e 0,886, respectivamente.

A análise fatorial da variável dependente da pesquisa, que se refere ao desempenho internacional, foi realizada de acordo com o nível de satisfação dos gestores em relação ao desempenho internacional, como demonstrado na Tabela 2. Nela, mantiveram-se as 12 variáveis oriundas do modelo original, que apresentaram cargas entre 0,769 e 0,901 e variância explicada de 71,466\%.

Ademais, a dimensão concernente ao desempenho internacional apresentou consistência interna adequada, ao denotar um Alpha de Cronbach no valor 0,961. 
Tabela 2 - Análise fatorial exploratória de desempenho internacional

\begin{tabular}{|c|c|c|c|c|c|}
\hline Dimensão & Variável & $\begin{array}{c}\text { Carga } \\
\text { fatorial }\end{array}$ & $\begin{array}{l}\text { Variância } \\
\text { explicada }\end{array}$ & KMO & $\begin{array}{l}\text { Alpha de } \\
\text { Cronbach }\end{array}$ \\
\hline \multirow{12}{*}{$\begin{array}{l}\text { Desempenho } \\
\text { internacional }\end{array}$} & D1 - Vendas internacionais. & 0,769 & \multirow{12}{*}{$71,466 \%$} & \multirow{12}{*}{0,817} & \multirow{12}{*}{0,961} \\
\hline & D2 - Crescimento das vendas internacionais. & 0,839 & & & \\
\hline & D3 - Rentabilidade internacional. & 0,885 & & & \\
\hline & $\begin{array}{l}\text { D4 - Retorno sobre investimento (ROI) dos } \\
\text { negócios internacionais. }\end{array}$ & 0,850 & & & \\
\hline & $\begin{array}{l}\text { D5 - Participação de mercado nos mercados } \\
\text { internacionais. }\end{array}$ & 0,849 & & & \\
\hline & D6 - Reputação internacional da empresa. & 0,774 & & & \\
\hline & $\begin{array}{c}\text { D7 - Introdução de novos produtos/serviços } \\
\text { em mercados internacionais. }\end{array}$ & 0,839 & & & \\
\hline & $\begin{array}{c}\text { D8 - Alcance global (presença em países } \\
\text { estrategicamente localizados ao redor do } \\
\text { mundo). }\end{array}$ & 0,774 & & & \\
\hline & $\begin{array}{l}\text { D9 - Tempo de comercialização de novos pro- } \\
\text { dutos/serviços em nível internacional. }\end{array}$ & 0,884 & & & \\
\hline & $\begin{array}{l}\text { D10 - Ganho de posições em mercados inter- } \\
\text { nacionais. }\end{array}$ & 0,876 & & & \\
\hline & $\begin{array}{c}\text { D11 - Número de novos produtos de sucesso } \\
\text { em mercados internacionais. }\end{array}$ & 0,901 & & & \\
\hline & D12 - Desempenho internacional. & 0,888 & & & \\
\hline
\end{tabular}

Fonte: dados da pesquisa.

\subsection{ANÁLISE UNIVARIADA - DESCRITIVA}

Tendo em vista que o instrumento de pesquisa adotado para o presente estudo é composto de variáveis medidas por escala Likert de 5 pontos, realizou-se a análise descritiva. Essa análise tem o objetivo de identificar o grau de importância de se possuir uma orientação de mercado voltada para o ambiente externo e habilidades de marketing internacional no processo de internacionalização, além de verificar a avaliação do desempenho internacional das empresas pesquisadas.

Com isso, identificou-se que a orientação para o mercado internacional, que consiste na capacidade de criar vantagem competitiva e em uma estratégia para o desenvolvimento de mercados internacionais (BARBOSA; AYALA, 2014), mostra-se de moderada importância para que as EBTs comercializem com o mercado externo, ao apresentar médias que variam entre 2,79 e 3,53 (Tabela 3).

Notou-se, também, que o comportamento da empresa de basear sua estratégia na compreensão das necessidades dos clientes internacionais apresenta-se como a melhor opção avaliada, ainda que de importância moderada. 
Tabela 3 - Análise descritiva de orientação para o mercado internacional

\begin{tabular}{|c|c|c|c|c|}
\hline Variáveis & Média & $\begin{array}{c}\text { Desvio-pa- } \\
\text { drão }\end{array}$ & Mínimo & Máximo \\
\hline $\begin{array}{l}\text { Resposta aos concorrentes internacionais } \\
\text { Se um concorrente lançou uma campanha intensiva } \\
\text { orientada para os nossos clientes internacionais, im- } \\
\text { plementamos uma resposta imediatamente. }\end{array}$ & 2,79 & 0,9679 & 1 & 5 \\
\hline $\begin{array}{l}\text { Integração das funções de negócio } \\
\text { Nossas funções de negócio (por exemplo, comercia- } \\
\text { lização/vendas, finanças e produção) são integradas } \\
\text { para atender às necessidades do mercado interna- } \\
\text { cional. }\end{array}$ & 3,19 & 1,1938 & 1 & 5 \\
\hline $\begin{array}{c}\text { Estratégia da empresa } \\
\text { A estratégia da empresa é baseada em nossa com- } \\
\text { preensão das necessidades dos clientes internacio- } \\
\text { nais. }\end{array}$ & 3,53 & 1,1198 & 1 & 5 \\
\hline $\begin{array}{l}\text { Satisfação dos clientes } \\
\text { A satisfação dos clientes internacionais com nossos } \\
\text { produtos ou serviços é medida sistematicamente. }\end{array}$ & 3,25 & 1,0544 & 1 & 5 \\
\hline
\end{tabular}

As habilidades de marketing são baseadas na maneira como as empresas geram conhecimento acerca do mercado para desenvolver capacidades de negócio (RIPOLLÉS; BLESA, 2012). Tendo em vista esse entendimento e com base nos dados apresentados na Tabela 4, verifica-se que essa habilidade tem moderada importância para que as EBTs se lancem ao mercado internacional.

Posto isso, percebe-se que, ao fazer uma comparação em termos de habilidades de marketing da situação da empresa em relação aos principais concorrentes, os participantes da pesquisa indicaram perceberem-se em uma situação mediana, ou seja, nem piores, nem melhores. Os resultados são expostos a seguir, na Tabela 4.

Tabela 4 - Análise descritiva de habilidades de marketing internacional

\begin{tabular}{|c|c|c|c|c|}
\hline Variáveis & Média & $\begin{array}{l}\text { Desvio-pa- } \\
\text { drão }\end{array}$ & Mínimo & Máximo \\
\hline $\begin{array}{l}\text { Processo de planejamento } \\
\text { Processo de planejamento de marketing internacional. }\end{array}$ & 2,89 & 0,8472 & 1 & 5 \\
\hline $\begin{array}{l}\text { Habilidades de segmentação e identificação } \\
\text { Habilidade para segmentar e identificar nichos de mer- } \\
\text { cado no exterior. }\end{array}$ & 3,08 & 0,9167 & 1 & 5 \\
\hline $\begin{array}{c}\text { Habilidades de uso de ferramentas de marketing } \\
\text { Habilidade para usar ferramentas de marketing para } \\
\text { diferenciar o produto (design, formação de preço e } \\
\text { publicidade). }\end{array}$ & 3,06 & 0,7946 & 1 & 5 \\
\hline $\begin{array}{l}\text { Desenvolvimento e adaptação de produto } \\
\text { Desenvolvimento e adaptação do produto no mercado } \\
\text { internacional. }\end{array}$ & 3,47 & 0,9528 & 1 & 5 \\
\hline Imagem da empresa & 3,15 & 0,8637 & 1 & 5 \\
\hline $\begin{array}{l}\text { Reconhecimento mundial } \\
\text { Reconhecimento mundial do produto/serviço. }\end{array}$ & 3,04 & 0,9798 & 1 & 5 \\
\hline
\end{tabular}
Fonte: dados da pesquisa. 
No que concerne ao desempenho internacional, a avaliação sustentou-se no que concerne ao nível de satisfação dos gestores em relação ao desempenho da empresa no mercado internacional. $O$ resultado obtido demonstra que tal avaliação se mostra em nível moderado, ao obter uma média de 3,27, como indica a Tabela 5.

Com os dados obtidos, observa-se que a variável melhor avaliada pelo prisma financeiro foi a de vendas internacionais, enquanto que, pelo ponto de vista operacional, foi a relacionada à reputação da empresa no mercado externo.

Tabela 5 - Análise descritiva da avaliação de desempenho internacional

\begin{tabular}{|c|c|c|c|c|}
\hline Variáveis & Média & $\begin{array}{l}\text { Desvio-pa- } \\
\text { drão }\end{array}$ & Mínimo & Máximo \\
\hline \multicolumn{5}{|c|}{ Desempenho financeiro } \\
\hline Vendas internacionais. & 3,42 & 1,2315 & 1 & 5 \\
\hline Crescimento das vendas internacionais. & 3,08 & 1,1742 & 1 & 5 \\
\hline Rentabilidade internacional. & 3,38 & 1,0043 & 1 & 5 \\
\hline $\begin{array}{l}\text { Retorno sobre investimento (ROI) dos negócios internacio- } \\
\text { nais. }\end{array}$ & 3,28 & 1,0447 & 1 & 5 \\
\hline \multicolumn{5}{|c|}{ Desempenho operacional } \\
\hline Participação de mercado nos mercados internacionais. & 3,04 & 1,1087 & 1 & 5 \\
\hline Reputação internacional da empresa. & 3,55 & 1,1859 & 1 & 5 \\
\hline $\begin{array}{l}\text { Introdução de novos produtos/serviços em mercados inter- } \\
\text { nacionais. }\end{array}$ & 3,28 & 1,0630 & 1 & 5 \\
\hline $\begin{array}{c}\text { Alcance global (presença em países estrategicamente loca- } \\
\text { lizados ao redor do mundo). }\end{array}$ & 3,08 & 1,2224 & 1 & 5 \\
\hline $\begin{array}{l}\text { Tempo de comercialização de novos produtos/serviços em } \\
\text { nível internacional. }\end{array}$ & 3,08 & 0,9167 & 1 & 5 \\
\hline Ganho de posições em mercados internacionais. & 3 & 1,0190 & 1 & 5 \\
\hline $\begin{array}{l}\text { Número de novos produtos de sucesso em mercados inter- } \\
\text { nacionais. }\end{array}$ & 3,02 & 0,9902 & 1 & 5 \\
\hline Desempenho internacional. & 3,13 & 1,0568 & 1 & 5 \\
\hline
\end{tabular}

Fonte: dados da pesquisa.

De maneira a prosseguir com os achados e a fim de testar a relação proposta pelas duas hipóteses deste estudo, no tópico seguinte são apresentados os resultados alusivos à análise de regressão.

\subsection{TESTE DE HOPÓTESE}

O teste de hipóteses foi realizado por meio da análise de regressão linear, com o intuito de averiguar a relação de dependência existente entre a variável dependente e a variável independente do estudo (HAIR et al., 2009). Para tal fim, as variáveis previsoras do modelo (independentes) consistem na orientação para o mercado internacional e nas habilidades de marketing, enquanto que a variável de resultado (dependente) é fixada pelo desempenho internacional, no que compete à sua avaliação. Ambas as variáveis foram obtidas a partir do cálculo da média ponderada dos fatores extraídos da análise fatorial.

Os resultados obtidos indicam um modelo de regressão formado pela inclusão da primeira variável independente e exclusão da segunda, com a consequente corroboração da $\mathrm{H}_{1}$ e refutação da $\mathrm{H}_{2}$. 
A Tabela 6, exposta a seguir, descreve os resultados do teste da primeira hipótese do estudo.

Tabela 6 - Análise de regressão linear - resultados

\begin{tabular}{|c|c|c|c|c|c|}
\hline Hipótese & Interação & $\mathbf{R}$ & $\mathbf{R}^{2}$ & $\mathbf{R}^{2}{ }_{a}$ & $\mathbf{p}$ \\
\hline $\mathrm{H}_{1}$ & $\begin{array}{c}\text { Orientação para o mercado internacional =>Desempenho } \\
\text { internacional }\end{array}$ & 0,504 & 0,255 & 0,238 & $* * *$ \\
\hline
\end{tabular}

Fonte: dados da pesquisa.

Com o exposto pela Tabela 6, conclui-se que o desempenho internacional é influenciado pela orientação para o mercado internacional em uma variabilidade de $23,8 \%$. Tal resultado demonstra que, quanto mais importante for a orientação para o mercado internacional na internacionalização das empresas pesquisadas, melhor será o desempenho internacional, corroborando, assim, a primeira hipótese do estudo $-\mathrm{H}_{1}$ : a orientação para o mercado internacional influencia positivamente o desempenho internacional.

Com base nisso, entende-se que possuir uma orientação de mercado voltada para o ambiente internacional desempenha forte contribuição para o desempenho, assim como apontado pelos achados de Maydeu-Olivares e Lado (2003), ao evidenciarem empiricamente que as organizações com maior orientação de mercado são capazes de obter melhores resultados do que os seus concorrentes. Nesse sentido, infere-se que as EBTs brasileiras que compõem a amostra deste estudo procuram fundamentar suas estratégias com base na compreensão do mercado internacional, ao mesmo tempo em que voltam sua atenção para os clientes e concorrentes em âmbito externo.

Por sua vez, a variável relacionada às habilidades de marketing internacional, por ser excluída do modelo de regressão, indicou não possuir relação de influência com o desempenho. Com tal resultado, refuta-se a segunda hipótese do estudo $-\mathrm{H}_{2}$ : a habilidade em marketing internacional influencia positivamente o desempenho internacional. Assim, infere-se que, com base nos resultados alcançados, as habilidades da empresa em criar valor para os clientes internacionais não influenciam o desempenho internacional.

\section{CONSIDERAÇÕES FINAIS}

Diante de constantes transformações comerciais e econômicas e da constante busca por maior competitividade, as empresas necessitam voltar sua atenção para seus aspectos mercadológicos, em especial no que tange à orientação de mercado e às habilidades de marketing, que, quando alocados em um contexto de atuação internacional, podem ser definidores do crescimento e da consolidação organizacional.

Tento em vista tal conjuntura, o presente estudo buscou analisar a relação de dependência entre a orientação para o mercado internacional e as habilidades de marketing internacional sobre o desempenho internacional das organizações. Para tal, realizou-se de um estudo quantitativo e descritivo, junto às EBTs brasileiras, com o intuito de alcançar o objetivo norteador da pesquisa e testar as hipóteses que o compõem.

Com tal esforço, obteve-se o retorno de 53 organizações localizadas em nove estados brasileiros (São Paulo, Rio Grande do Sul, Santa Catarina, Minas Gerais, Rio de Janeiro, Paraná, Pernambuco, Paraíba e Bahia) e no Distrito Federal. A partir da execução do teste de hipótese, realizado mediante a efetivação da análise de regressão linear, obteve-se um modelo composto pela existência de relação entre a orientação para o mercado internacional e o desempenho internacional, enquanto que as variáveis referentes às habilidades de marketing internacional 
foram excluídas do modelo, demonstrando não possuir influência sobre tal desempenho.

A presente pesquisa, ao enfocar a temática de desempenho internacional, soma-se aos estudos mais recentes acerca de negócios internacionais, que buscam entender os aspectos que envolvem sua avaliação. $\mathrm{E}$, diante dos resultados alcançados, contribui com a aplicação de um instrumento de pesquisa quantitativo referente à avaliação do desempenho internacional que ainda não havia sido testado no contexto brasileiro. Tal instrumento, elaborado com base no modelo desenvolvido por Gerschewski, Rose e Lindsay (2015), mostrou atender de forma adequada às características do citado ambiente e apresentar consistência interna para responder ao objetivo proposto.

Todavia, ainda que os resultados sejam capazes de atender ao objetivo proposto, atenta-se para a principal limitação desta pesquisa, centrada no feito de as evidências obtidas referirem-se apenas à realidade das empresas que compuseram a amostra investigada, o que impossibilita a extrapolação dos resultados. Ademais, sugere-se que novos estudos sejam realizados com o intuito de atentar para o entendimento dos influenciadores e determinantes de desempenho, como forma de verificar os fatores organizacionais e ambientais que gerem influências sobre o desempenho organizacional e a consolidação das empresas no mercado externo.

\section{REFERÊNCIAS}

AUH, S.; MERLO, O. The power of marketing within the firm: it's contribution to business performance and the effect of power asymmetry. Industrial Marketing Management, v. 41, n. 5, p. 861-873, jul. 2012.

BARBOSA, D. M. E.; AYALA, A. H. LOS determinantes de la orientación exportadora y los resultados en las pymes exportadoras en Colombia. Estudios Gerenciales, Cali, v. 30, n. 133, p. 430-440, out./dez. 2014.

BRETTEL, M.; ENGELEN, A.; HEINEMANN, F. New entrepreneurial ventures in a globalized world: the role of market orientation. Journal of International Entrepreneurship, Nova York, v. 7, n. 2, p. 88-110, jun. 2009.

CARNEIRO, J.; BRENES, E. R. Latin American firms competing in the global economy. Journal of Business Research, Amsterdã, v. 67, n. 5, p. 831-836, mai. 2014.

CAVUSGIL, S. T.; SHAOMING, Z. Marketing strategy-performance relationship: An investigation of the empirical link in export market ventures. Journal of Marketing, Chicago, v. 58, n.1, p. 1-21, jan. 1994.
DALMORO, M. Estratégias de marketing internacional e a relação com a performance exportadora e orientação para mercado externo. Internext - Revista Eletrônica de Negócios Internacionais da ESPM, São Paulo, v. 4, n. 2, p. 1-18, jul./dez. 2009.

DAY, G. S. The capabilities of market-driven organizations. Journal of Marketing, Chicago, v. 58, n. 4, p. 37-52, out. 1994.

DONG, X. D.; ZHANG, Z.; HINSCH, C. A.; ZOU, $S$. Reconceptualizing the elements of market orientation: a process-based view. Industrial Marketing Management, v. 56, p. 130-142, jul. 2016.

ENGELMAN, R., ZEN, A. C.; FRACASSO, E. $M$. The impact of the incubator on the internationalization of firms. Journal of Technology Management \& Innovation, v. 10, n. 1, mar. 2015.

FILATOTCHEV, I.; LIU, X.; BUCK, T. Y.; WRIGHT, $M$. The export orientation and export performance of high-technology SMEs in emerging markets: the effects of knowledge transfer by returnee entrepreneurs. Journal of International Business Studies, East Lansing, v. 40, n. 6, p. 1005-1021, ago. 2009. 
FLEURY, A.; FLEURY, M. T. L. Local enablers of business models: the experience of brazilian multinationals acquiring in north america. Journal of Business Research, v. 67, n. 4, p. 516-526, abr. 2014

GARCÍA, A. N.; GONZÁLEZ, F. J. A.; PÉREZ, F. L.; SANMARTÍN, E. R. Efectos de la proactividad exportadora y la orientación al mercado en las operaciones de comercio exterior. Revista Española de Investigación de Marketing ESIC, Madrid, v. 16, n. 1, p. 113-133, mar. 2012.

GERSCHEWSKI, S.; ROSE, E. L.; LINDSAY, V. J. Understanding the drivers of international performance for born global firms: an integrated perspective. Journal of World Business, v. 50, n. 3, p. 558-575, jul. 2015.

GNIZY, I. Power dynamics of the international marketing within firms and how they shape international performance. Industrial Marketing Management, v. 57, p. 148-158, ago. 2016.

HAIR, J. F. et al. Análise multivariada de dados. 6 ed. São Paulo: Bookman, 2009.

$\mathrm{HE}, \mathrm{X}$; WEI, Y. Linking market orientation to international market selection and international performance. International Business Review, v. 20, n. 5, p. 535-546, out. 2011.

HOMBURG, C.; VOMBERG, A.; ENKE, M.; GRIMM, P. H. The loss of the marketing department's influence: is it really happening? And why worry? Journal of the Academy Marketing Science, Gewerbestrasse, v. 43, n. 1, p. 1-13, jan. 2015.

JAWORSKI, B. J.; KOHLI, A. K. Orientação para o mercado: antecedentes e consequências. Revista de Administração de Empresas, São Paulo, v. 46, n.2, abr./jun. 2006.

KNIGHT, G.A.; CAVUSGIL, S.T. Innovation, organizational capabilities, and the born global firm. Journal of International Business Studies, East Lansing, v. 35, n. 2, p. 124-141, mar. 2004.

KNIGHT, G. A.; KIM, D. International business competence and the contemporary firm. Journal of International Business Studies, East Lansing, v. 40, n. 2, p. 255-273, fev./mar. 2009.

KOHLI, A. K.; JAWORSKI, B. J. Market orientation: the construct, research propositions, and managerial implications. Journal of Marketing, v. 54, n. 2, p. 1-18, abr. 1990.

LI, L.;QIAN,G.; QIAN,Z. Earlyinternationalization and performance of small high-tech born globals. International Marketing Review, Bradford, v. 29, n. 5, p. 536-561, 2012.

LIAO, S.-H.; CHANG, W.-J.; WU, C.-C.; KATRICHIS, J. M. A survey of market orientation research (1995- 2008). Industrial Marketing Management, v. 40, n. 2, p. 301-310, fev. 2011.

LINDON, D.; LENDREVIE, J.; RODRIGUES, J. V.; DIONÍSIO, P. Mercator 2000: Teoria e prática do marketing. 9 ed. Lisboa: Publicações Dom Quixote, 2000.

MALHOTRA, N.K. Pesquisa de marketing: uma orientação aplicada. 3 ed. Porto Alegre: Bookman, 2001.

MAYDEU-OLIVARES, A.; LADO, N. Market orientation and business economic performance, a mediated model. International Journal of Service Industry Management, v. 14, n. 3, p. 284-309, 2003.

MORAIS, I. D. C.; SOARES, A. M. Impacto do marketing interno sobre a orientação para o mercado em empresas brasileiras. Revista de Administração Contemporânea, Rio de Janeiro, v. 20, n. 2, art. 4, p. 197-215, mar./abr. 2016.

MOURA, M. L. C.; HONÓRIO, L. C. Características da firma, marketing internacional e desempenho exportador: um survey com exportadoras mineiras. Revista Alcance Eletrônica, Itajaí, v. 19, n. 2, p. 165-181, abr./ jun. 2012. 
NARVER, J. C.; SLATER, S. F.; MACLANCHLAN, D. L. Total market orientation, business performance, and innovation. Marketing Science Institute, Cambridge, 2000. Disponível em: < http://www.msi.org/reports/totalmarket-orientation-business-performanceand-innovation/>. Acesso em: 15 jan. 2016.

NETO, J. F. R.; GALLEGO, P. A. M.; SOUZA, C. C.; RODRIGUES, W. O. P. O papel da orientação empreendedora no relacionamento entre orientação para o mercado e desempenho empresarial: evidências das pequenas empresas do comércio. Revista Eletrônica de Administração, Porto Alegre, v. 74, n.1, p. 115138, jan./abr. 2013.

PAIM, N. A.; LARA, J. E.; CHRISTINO, J. M. M. Relações quantitativas entre orientação para o mercado, desempenho organizacional e a percepção externa de sucesso em instituições de pesquisas tecnológicas afiliadas à ABIPTI. Revista de Administração Contemporânea, Curitiba, v. 15, n. 6, art. 5, p. 1059-1077, 2011.

PALACIOS, T. M. B.; SOUSA, J. M. M.. Estratégias de marketing internacional. São Paulo: Atlas, 2004.

RIBEIRO, F. C. F. Born Globals brasileiras: estudo da internacionalização de empresas de base tecnológica. 2012. 229 f. Tese (Doutorado) Universidade de São Paulo. São Paulo, 2012.

RIPOLLÉS, M.; BLESA, A. International new ventures as "small multinationals": the importance of marketing capabilities. Journal of World Business, v. 47, n. 2, p. 277-287, abr. 2012.

SONG, M.; NASON, R.; BENEDETTO, C. Distinctive Marketing and Information Technology Capabilities and Strategic Types: A cross-national investigation. Journal of International Marketing, Chicago, v. 16, n. 1, p. 4-38, mar. 2008.

TAPSCOTT, D.; CASTON, A. Mudança de paradigma. São Paulo: Makron Books, 1995.
ZAHRA, S. A; IRELAND, R. D, HITT, M. A. H. International expansion by new venture firms: international diversity, mode of market entry, technological learning, and performance. The Academy of Management Journal, v. 43, n. 5, p. 925-950, out. 2000.

ZOU, S.; CAVUSGIL, S. T. The GMS: a broad conceptualization of global marketing strategy and its effect on firm performance. Journal of Marketing, v. 66, n. 4, p. 40-56, out. 2002. 